\title{
Excitation Frequencies of the Effects of Selective Drift of Solvated Cations in Moving Salts Solution
}

\author{
Igor Shamanin ${ }^{1}$, Mishik Kazaryan ${ }^{2,3}$, Valeriy Gofman ${ }^{1}$, Sergey Timchenko ${ }^{1,},{ }^{*}$, Andrei Poberezhnikov ${ }^{1}$, Ilya Tuksov $^{1}$ \\ ${ }^{1}$ National Research Tomsk Polytechnic University, Tomsk, Russian Federation \\ ${ }^{2}$ Lebedev Physical Institute of the Russian Academy of Sciences, Moscow, Russian Federation \\ ${ }^{3}$ National Research Tomsk state University, Tomsk, Russian Federation
}

\begin{abstract}
Interaction of external electric field with free and bound charges in the salt solution volume in polar dielectric fluid was considered and the equation describing ion oscillations relative to solvent molecules was obtained. Fundamental frequencies of the solvation shell relative to the ion in different approximations describing the system: ion - solvation shell were evaluated. The results of comparison with the experiment pointed out that the model, in which the solvated ion is considered as a spherical rotator formed by the solvent molecules layer bordering with the external solvation shell, is the most appropriate. It provides an opportunity of development of new technologies for extraction of rare earth elements.
\end{abstract}

\section{Introduction}

Experimental data and theoretical estimations [1] have shown, that excitation of rotary-onward motions of solvated cations (clusters) with various inertial properties can become a basis for technology of separation of rare earth elements in water solutions of their salts. Practical use of the discovered phenomenon of metals solvated cations selective field-assisted motion in solutions of salt mixtures, influenced by external periodic electric field, will require the presence of some relations applicable for engineering calculations. In particular, to determine velocity of motion of the solvated ion under direct influence of external electrical field the parameters of its oscillatory motion caused by that field need to be defined. The solvated ion is in a polar dielectric fluid and the electric field influences both the ion and the solvent molecules. In its turn, the internal electric field influences the solvent molecules surrounding the ion. The task of determining the equation describing ion oscillations relating to the surrounding solvent molecules is not trivial.

To determine such equation, interrelation of electric fields and currents corresponding to some charge distribution in the salt solution in polar dielectric fluid should be considering.

\section{External electric field influences on free and bound charges in solution}

Electric field intensity distribution $\vec{E}(\vec{r})$ and volume charge density distribution $\rho(\vec{r})$ are connected by Poisson's equation [2]:

$$
\operatorname{div} \vec{E}(\vec{r})=4 \pi \rho(\vec{r}) .
$$

If salt, the molecules of which are dissociated, is dissolved in dielectric fluid, the electric field in the fluid volume is created by free charge carriers - ions formed at dissociation with the volume charge density distribution $\rho_{f}(\vec{r})$. This field intensity distribution is also described by Poisson's equation:

$$
\operatorname{div} \vec{E}_{f}(\vec{r})=4 \pi \rho_{f}(\vec{r}) .
$$

The molecules of dielectric fluid (solvent) polarize in the electric field of free charge carriers. So the following condition is fulfilled [3]:

$$
\operatorname{div}(\vec{E}(\vec{r})+4 \pi \vec{P}(\vec{r}))=4 \pi \rho(\vec{r}),
$$

where $\vec{P}(\vec{r})$ - polarization density distribution. Moreover, this condition is fulfilled, no matter how $\vec{E}(\vec{r})$ and $\vec{P}(\vec{r})$ is connected with each other.

When the external electric field is applied to the solution, it results in its local electrical neutrality breaking, that is, volume charge density other than zero appears. Besides, these charges are connected and appear entirely due to non-uniform polarization of dielectric fluid molecules. Volume density distribution of the bound charges $\rho_{b}(\vec{r})$ is determined by the relation:

$$
\rho_{b}(\vec{r})=-\operatorname{div} \vec{P}(\vec{r}) .
$$

In the general case the volume charge consists of two components - free charges density and bound charge density, which appear when there is non-uniform polarization of the sample $\vec{P}(\vec{r})$.

Consideration of one-dimensional case (when the solution is in the form of a flat slab, the transverse sizes of which are much greater than its thickness) can be confined for simplicity. The polarization density is

\footnotetext{
* Corresponding author: timsn@tpu.ru
} 
connected with the external electric field intensity $\vec{E}_{0}$ created by plan-parallel electrodes isolated from the solution by the relation $4 \pi \vec{P}=\vec{E}_{0}-\vec{E}$,

where $\vec{E}$ is the field intensity inside the layer of the solution located between the electrodes.

The electric field intensity inside the solution is distributed in a particular way, that is, it depends on the coordinate. At that, the averaged intensity value in the solution volume $\langle E\rangle$ is smaller than the filed intensity between the electrodes $E_{0}$. The ratio $E_{0} /\langle E\rangle$ can be evaluated using the equation (1) and the correlation between the polarization density and intensity of the field causing dielectric fluid polarization:

$$
\vec{P}=\frac{3}{4 \pi}\left(\frac{\varepsilon-1}{\varepsilon+2}\right) \vec{E},
$$

where $\varepsilon$ - dielectric permeability of the solvent.

If the electric field is created by the charge with the volume density $\rho$, and polarization is caused by the electric field of this charge, the equation (1) can be rewritten in the following form:

$$
\left(1+3\left(\frac{\varepsilon-1}{\varepsilon+2}\right)\right) \operatorname{div} \vec{E}=4 \pi \rho \text {. }
$$

This equation shows that when the charge density $\rho$ is the same, polarization causes $\left(1+3\left(\frac{\varepsilon-1}{\varepsilon+2}\right)\right)$ times decrease of the electric field intensity. When dielectric permeability has greater values $(\varepsilon>10)$, the field intensity in the dielectric fluid volume $\langle E\rangle$ is approximately 4 times smaller than the intensity between the electrodes $E_{0}$. When the values of dielectric permeability are close to 1 , the field intensity in the dielectric fluid volume $\langle E\rangle$ is approximately 2 times smaller than the intensity between the electrodes $E_{0}$.

If the electric field intensity distribution is considered, it follows from the mentioned above that the intensity increases in the plane solution layer volume from the value $\langle E\rangle$ to the value $E_{0}$ at the approach to the electrodes isolated from the solution. Macroscopic value of the bound charges density $\rho_{b}(\vec{r})$ will be not equal to zero in these solution regions close to the electrodes, because moving from the electrodes the field intensity will be weakly depend on the coordinate and will have the value $\langle E\rangle \approx$ const. Also the polarization density $\vec{P}$ will not change significantly and $\operatorname{div} \vec{P}(\vec{r})$ will be close to zero. Consequently, the macroscopic value of bound charges density $\rho_{b}(\vec{r})$ by virtue of the equation (2), tends to zero while moving away from the electrodes.

Taking into consideration the above presented reasoning it is possible to write the equation connecting electric fields intensities in the solution volume with the free charge density in the saline solution in the dielectric fluid:

$\operatorname{div}\left(\vec{E}_{f}(\vec{r})+3\left(\frac{\varepsilon-1}{\varepsilon+2}\right)\left(\vec{E}_{0}(\vec{r})+\vec{E}_{f}(\vec{r})\right)\right)=4 \pi \rho_{f}(\vec{r})$.

The equation for the solution layer which is between two plane electrodes isolated from the solution will have the following form:

$\frac{\partial}{\partial x}\left(E_{f}(x)+3\left(\frac{\varepsilon-1}{\varepsilon+2}\right)\left(E_{0}(x)+E_{f}(x)\right)\right)=4 \pi \rho_{f}(x)$,

or, which is the same, as:

$\left(1+3\left(\frac{\varepsilon-1}{\varepsilon+2}\right)\right) \frac{\partial E_{f}(x)}{\partial x}+3\left(\frac{\varepsilon-1}{\varepsilon+2}\right) \frac{\partial E_{0}(x)}{\partial x}=4 \pi \rho_{f}(x)$.

\section{Ion oscillation equation relative to solvent molecules}

Differentiate the obtained equation with respect to time:

$$
\begin{aligned}
& \left(1+3\left(\frac{\varepsilon-1}{\varepsilon+2}\right)\right) \frac{\partial}{\partial t}\left(\frac{\partial E_{f}(x)}{\partial x}\right)+ \\
& 3\left(\frac{\varepsilon-1}{\varepsilon+2}\right) \frac{\partial}{\partial t}\left(\frac{\partial E_{0}(x)}{\partial x}\right)=4 \pi \frac{\partial \rho_{f}(x)}{\partial t}
\end{aligned}
$$

The right member of this equation is none other than the current density $j_{i o n}$ of free charge carriers (ions) multiplied by $4 \pi$ :

$$
4 \pi \frac{\partial \rho_{f}(x)}{\partial t}=4 \pi j_{i o n}(t) .
$$

For a single ion located in the solution the space intensity distribution of the electric field created by it can be defined in a functional form:

$$
E_{f}(x)=k q_{\text {ion }} \frac{x-x_{0}(t)}{\left|x-x_{0}(t)\right|^{3}},
$$

where $k$ - invariable, $q_{\text {ion }}$ - ion electric charge, $x_{0}(t)$ - ion coordinate, which changes with respect to time due to ion motion in the external electric field. Then:

$$
\frac{\partial}{\partial t}\left(\frac{\partial E_{f}(x)}{\partial x}\right)=k q_{i o n} \frac{\partial}{\partial t}\left(\frac{1}{\left|x-x_{0}(t)\right|^{3}}-3 \frac{1}{\left(x-x_{0}(t)\right)^{3}}\right)
$$

and

$\frac{\partial}{\partial t}\left(\frac{\partial E_{f}(x)}{\partial x}\right)=-6 \frac{k q_{\text {ion }}}{\left(x-x_{0}(t)\right)^{4}} \frac{\partial x_{0}(t)}{\partial t}=-6 \frac{k q_{\text {ion }}}{\left(x-x_{0}(t)\right)^{4}} v_{\text {ion }}(t)$, where $v_{i o n}(t)=\frac{\partial x_{0}(t)}{\partial t}$ - the directed ions motion velocity.

Taking into consideration that the ions current density is $j_{i o n}(t)=n q_{i o n} v_{i o n}(t)$, where $n$ - the ions density, the equation (3) for the coordinate of the single ion $x_{i}(t)$, located in the salt solution in the polar dielectric fluid with great dielectric permeability, can be written as follows:

$$
-24 \frac{k q_{\text {ion }}}{\left(x-x_{i}(t)\right)^{4}} \frac{\partial x_{i}(t)}{\partial t}+3 \frac{\partial}{\partial t}\left(\frac{\partial E_{0}(x)}{\partial x}\right)=4 \pi n q_{i o n} \frac{\partial x_{i}(t)}{\partial t},
$$

or 


$$
\left(4 \pi n+24 \frac{k}{\left(x-x_{i}(t)\right)^{4}}\right) q_{i o n} \frac{\partial x_{i}(t)}{\partial t}=3 \frac{\partial}{\partial t}\left(\frac{\partial E_{0}(x)}{\partial x}\right) .
$$

The numerical value of the invariable $k$ (the proportionality factor in the coulomb law) in the system SI of physical quantities is $k \approx 9 \cdot 10^{9}$. Let us choose the fixed point in the solution volume $x=a$, in which the gradient of the field intensity is known, and its time dependence is $\left(\frac{\partial E_{0}(a)}{\partial x}\right)=f(t)$. In the experiments on excitation of the field-assisted motion of solvated ions [4] $f(t) \approx A(1+2 \sin \omega t-\sin 2 \omega t)$, the invariable value $A \approx 4 \cdot 10^{6} \mathrm{~V} \cdot \mathrm{m}^{-2}$. The ion oscillation amplitude influenced by the external electric field $\left(a-x_{i}(t)\right)_{\max }$ certainly does not exceed $10^{-3} \mathrm{~m}$, thus, the first part within the brackets of the left side of the equation can be neglected. Therefore, the equation of ion oscillation $\Delta(t)$ can be written as follows:

$$
\begin{aligned}
& 24 k q_{i o n} \frac{1}{\Delta(t)^{4}} \frac{\partial}{\partial t} \Delta(t)=6 A \omega(\cos \omega t-\cos 2 \omega t), \\
& \text { or } \\
& \frac{1}{\Delta(t)^{4}} \frac{\partial}{\partial t} \Delta(t)=\frac{A \omega}{4 k q_{\text {ion }}}(\cos \omega t-\cos 2 \omega t) .
\end{aligned}
$$

The solution of the equation (4) takes the form [5]:

$$
\Delta(t)=\left(\frac{A}{8 k q_{\text {ion }}}(\sin 2 \omega t-2 \sin \omega t)+C\right)^{-1 / 3},
$$

where the invariable $C$ is determined from the condition $\Delta(t=0)=a$.

The oscillation frequency $v=\frac{\omega}{2 \pi}$ is the same as the frequency of the external electric field and is the oscillation frequency of the single ion relative to the solvation shell. If this frequency is coincident with the fundamental frequency of the solvated ion (the system: the ion - the solvation shell), the transition of oscillative motion into translational field-assisted motion of the solvated ion should be expected.

\section{Solvated ion fundamental frequencies}

\subsection{Solvated ion (cluster) as a liquid droplet}

Consider the cluster formed by the central ion and the surrounding associated solvent molecules as a liquid droplet. The fundamental frequencies for the droplet oscillation relative to static equilibrium position on the assumption of the minuteness of velocities field perturbation inside the droplet and free surface can be defined by the formula [6]:

$$
v_{l}^{2}=\frac{\sigma_{s}}{\rho R_{0}^{3}} l(l-1)(l+2), l=2,3, \ldots,
$$

where $\rho$ - fluid density, $\sigma_{s}$ - surface tension coefficient, $R_{0}$ - droplet (cluster) radius.
In order to define the surface tension coefficient it is necessary to evaluate the work to be done to create the surface limiting the cluster. Coming out of the solvent molecule to this surface leads to increase of the cluster energy by the binding energy of the solvent molecule inside the cluster. The surface tension coefficient will be equal to the ratio of this energy $\varepsilon_{b}$ to the area of the sphere surface $s$, falling on one solvent molecule:

$$
\sigma_{s}=\frac{\varepsilon_{b}}{s} .
$$

The cluster radius is determined from the condition of equality of critical electric field intensity to the intensity value near the central ion [1]. In Figure 1 the distributions $E_{r}(r)$ for different ions in water are presented.

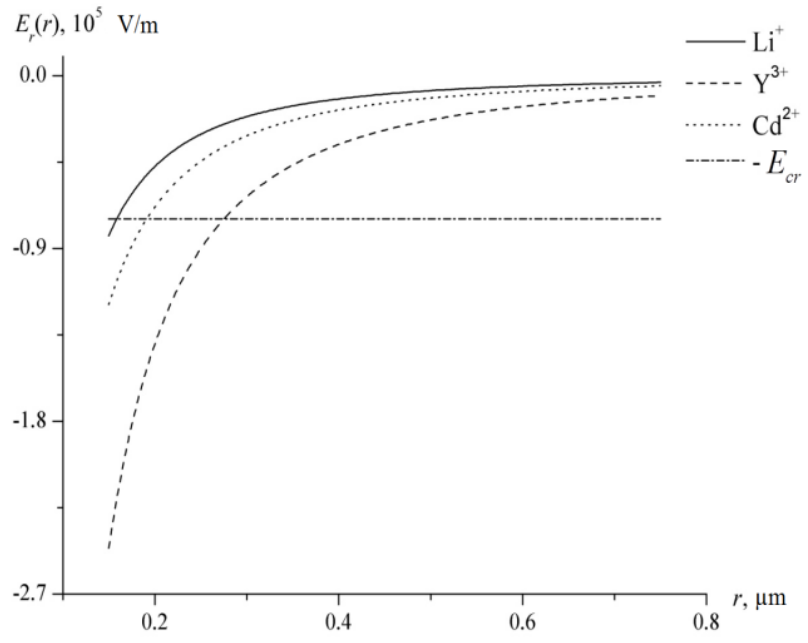

Fig. 1. Dependencies $E_{r}(r)$ for cations $\mathrm{Li}^{+}, \mathrm{Cd}^{2+}, \mathrm{Y}^{3+}$ for the temperature of $298 \mathrm{~K}$ and critical electric field intensity $E_{c r}$.

Consider that the cluster radius $R_{0}$ is equal to $0.2 \mu \mathrm{m}$ ( $\left.E_{r}(r)=E_{c r}\right)$ for evaluation. At usual conditions $9 \cdot 10^{5}$ molecules of water can be located within the sphere with the radius $R_{0}$. The sphere surface area is $50.24 \cdot 10^{-14} \mathrm{~m}^{2}$, and $s \approx 5.55 \cdot 10^{-19} \mathrm{~m}^{2}$ falls on one molecule.

To determine the binding energy of the solvent molecule inside the cluster it is possible to assume that it is equal to the dipole binding energy in the central ion electric field:

$$
\varepsilon_{b} \sim p \cdot E,
$$

where $p$ - intrinsic dipole moment of the solvent molecule, $E$ - electric field intensity within the cluster radius (see Figure 1). The binding energy $\varepsilon_{b}$ for the intrinsic dipole moment of the water molecule $p=$ $6.2 \cdot 10^{-30} \mathrm{C} \cdot \mathrm{m}$ will be about $10^{-24} \mathrm{~J}$. The surface tension coefficient will be $\sigma_{s} \approx 2 \cdot 10^{-6} \mathrm{~J} / \mathrm{m}^{2}$. Therefore, the intrinsic oscillation frequency of the cluster as a droplet can reach several hundreds of $\mathrm{kHz}$. It is equal to the hydrogen binding energy in the solvent which falls on one solvent molecule. The hydrogen binding energy in water is $21 \mathrm{~kJ} / \mathrm{mole}$, consequently, $\varepsilon_{b} \approx 3.5 \cdot 10^{-20} \mathrm{~J}$ falls on one solvent molecule. The surface tension coefficient 
is $\sigma_{s} \approx 0.063 \mathrm{~J} / \mathrm{m}^{2}$. Then the intrinsic oscillation frequency of the cluster as a droplet is tens of $\mathrm{MHz}$.

\subsection{Solvated ion (cluster) as a spherical pendulum}

Consider the cluster formed by the central ion and the surrounding associated solvent molecules as a hollow sphere with the inner radius $R_{1}$, the outer radius $R_{2}$ and the mass $M$. In this case the hollow sphere can rotate about the axis passing through its geometric center. The sphere is connected with the axis by elastic spiral spring with the tension $k$, which provides the sphere with a steady state position. In this model the cluster is considered as a torsional spherical pendulum. The spring tension is determined by the binding energy of the central ion with the solvent molecules located at some distance from it. These molecules form the first solvation radius $R_{1}$, and their amount is equal to the ion coordination number in the solvent.

The fundamental oscillations frequency of such a pendulum is defined by the formula:

$$
v=\sqrt{\frac{k}{J}},
$$

where $J$ - moment of pendulum inertia the value of which can be defined by the formulae:

$$
\begin{aligned}
& J=\frac{2}{5} M R_{2}^{2}-\text { for } R_{2} \gg R_{1} ; \\
& J=\frac{2}{3} M\left(\frac{R_{1}+R_{2}}{2}\right)^{2} \text { - for } R_{2} \sim R_{1} .
\end{aligned}
$$

The spring tension is defined from breakeven condition of electrostatic interaction of the central ion with polarized solvent molecules located at the first solvation radius, and the spring tension energy:

$$
k\left(R_{1}-r_{i}\right)=E \cdot n \cdot q,
$$

where $r_{i}$ - central ion radius, $n$ - solvent molecules amount, $q$-partial charge which is obtained by the solvent molecule in the ion electric field.

For the ion $\mathrm{Ca}^{2+}$ the radius $r_{i}=0.114 \cdot 10^{-9} \mathrm{~m}$, when the coordination number is $n=6$, and $r_{i}=0.148 \cdot 10^{-9} \mathrm{~m}$, when the coordination number is $n=12$. As this takes place, the first solvation radius will be $R_{1}=0.229 \cdot 10^{-8} \mathrm{~m}$ for $n=6$ and $R_{1}=0.324 \cdot 10^{-8} \mathrm{~m}$ for $n=12$. Supposing that the outer radius $R_{2}$ is determined from the condition $E_{r}\left(R_{2}\right)=E_{c r}$ and is equal to $0.2 \mu \mathrm{m}$, then, the mass of the spherical torsional pendulum will be $M \approx 33.5 \cdot 10^{-18}$ $\mathrm{kg}$. If the solvent is water, water molecules will be oriented in such a way that oxygen atoms will be located closer to the central ion $\mathrm{Ca}^{2+}$. By virtue of significant difference in electronegativity of hydrogen and oxygen the oxygen atom in the water molecule acquires significant negative partial charge $q=-0.66 e$. As a result the tension value for the solvated ion $\mathrm{Ca}^{2+}$ will be $k=(45 \ldots 70) \cdot 10^{-6} \mathrm{~N} / \mathrm{m}$. So the natural oscillation frequency of the cluster as a torsional spherical pendulum will be about $10 \mathrm{THz}$.

\subsection{Solvated ion (cluster) as a spherical rotator}

Consider the cluster formed by the central ion and the surrounding associated solvent molecules as a hollow sphere with the inner radius $R_{1}$, outer radius $R_{2}$ $\left(R_{2}>>R_{1}\right)$ and the mass $M$. Insider the hollow spherical shell there is the central ion.

The sphere volume is rather small compared to the cluster volume having the radius $R_{2}$. However, the hollow sphere can rotate about the mass center of the cluster which practically aligns with the spherical shell geometrical center, because the shell mass $M$ is significantly more than the central ion $\operatorname{mass} m_{i}$. Therefore, natural frequencies for such a system can be defined by the formula:

$$
v_{l}=\frac{h}{8 \pi^{2} J} l(l+1), l=1,2,3, \ldots,
$$

where $h$ - Planck's constant, $J=M R_{2}^{2}$ - inertia moment of the rotator.

Depending on the value $R_{2}$, which in its turn defines the value $M$, natural frequencies change from units of $\mathrm{Hz}$ for the cluster with the radius $R_{2} \sim 0.1 \mu \mathrm{m}$ up to tens of $\mathrm{Hz}$ for the cluster the solvation shell of which is formed by 12 water molecules.

\section{Conclusion}

1. The equation of the ion oscillations in the solution for the case of "asymmetric" electric field is determinated. A mathematical model, which allows a better understanding of the process of separation of the solvated ions under the influence of an electric field and calculate required frequency for excitation of selective drift effect of cations of different chemical elements, is created.

2. Fundamental oscillation frequencies of the ion in the salt solution in polar dielectric fluid are within the interval from tens of $\mathrm{Hz}$ to units of $\mathrm{kHz}$ and correspond to two possible models of the solvated ion:

a) the solvated shell mass does not exceed the value of $10^{-22} \mathrm{~kg}$, the outer radius of the shell is not bigger than $0.05 \mu \mathrm{m}$, which is by an order of magnitude greater than the first solvation radius. When the solvent is water, about $10^{4}$ water molecules are within the solvation shell. And the fundamental oscillations of the rotator will be tens of $\mathrm{Hz}$;

b) only relatively mobile molecules of the solvent which are not closely connected by the central ion electric field and border to the surface with the radius $R_{2}$ take part in the rotational motion. The value $R_{2}$ is defined from the condition $E_{r}\left(R_{2}\right)=E_{c r}$. The total 
amount of such mobile molecules is significantly smaller than the number of the solvent molecules associated around the central ion. The mass of such a spherical rotator, formed by the layer of the solvent molecules fitting to the external border of the solvation shell, does not exceed $10^{-23} \mathrm{~kg}(350 \ldots 400$ water molecules). As this takes place, the fundamental frequencies of the rotator are units of $\mathrm{kHz}$.

On the example of yttrium it is shown the possibility of the obtained results using as a basis for technology of rare earth elements separation in water solutions of their salts.

\section{References}

1. M.A. Kazaryan, I.V. Lomov, I.V. Shamanin, Electrophysics of Structurized Saline Solutions in Fluid Polar Dielectrics (Fizmatlit, Moscow, 2011)

2. R.F. Feynman, Feynman Lectures on Physics: transl. from English (Mir, Moscow, 1976)

3. A. Parcell, Berkeley Course of Physics: transl. from English: In $5 \mathrm{Vol}$ - $-3 \mathrm{rd}$ revised edition. Vol. 2: Electricity and Magnetism (Nauka, Moscow, 1983)

4. M.A. Kazaryan, I.V. Shamanin, I.V. Lomov, Theoretical Basis of Chem. Technol. 44, 1, 1 (2010)

5. E. Kamke, A guide on ordinary differential equations: transl. from German (5th edition, Nauka, Moscow, 1976)

6. I.A. Lukovskiy, M.A. Chernova, Akusticheskij Vestnik, 14, 3, 23 (2011) 